\title{
Expecting dirt but saying dart: The creation of a blend memory
}

\author{
MICHAEL S. HUMPHREYS, JENNIFER S. BURT, and SANDRA LAWRENCE \\ University of Queensland, Brisbane, Queensland, Australia
}

\begin{abstract}
During a naming task, time pressure and a manipulation of the proportion of related prime-target pairs were used to induce subjects to generate an expectation to the prime. On some trials, the presented target was orthographically and generally phonologically similar to the expected target. The expectancy manipulation was barely detectable in the priming data but was clearly evident on a final recognition test. In addition, the recognition data showed that the nearly simultaneous activation of an expectation and sensory information derived from the orthographically and phonologically similar target produced a false memory. It is argued that this represents a blend memory.
\end{abstract}

Semantic priming in the lexical decision or wordnaming task is revealed when responses to the target word (a yes response in lexical decision and the pronunciation of the word in naming) are faster when the target is immediately preceded by a related (generally, an associate) word. Semantic priming is revealed in the perceptual identification task in which a degraded target word that is related to its prime is more likely to be identified correctly than is a degraded target word that is unrelated to its prime. Although semantic priming effects in all three tasks are generally similar, there are some differences (Neely, 1991; Seidenberg, Waters, Sanders, \& Langer, 1984). Prominent among these differences has been the response to the proportion of related prime-target pairs. In lexical decision, responses to related targets become faster as the proportion of related prime-target pairs increases (Tweedy, Lapinski, \& Schvaneveldt, 1977). Similarly, in perceptual identification, the probability of correctly identifying a related target increases as this proportion increases (Burt, Walker, Humphreys, \& Tehan, 1993). However, for some years, it was thought that the relatedness proportion effect did not occur in the naming task. This lack of an effect was cited as evidence that expectancy strategies (the subject uses the prime to predict the target or a set of items that might contain the target) were not used in naming (Seidenberg et al., 1984).

It is now known that a relatedness proportion effect can be found in naming when the variation in relatedness proportion is large (Burt, Mardle, \& Humphreys, 1996; Keefe $\&$ Neely, 1990). Thus, there is evidence for the use of an expectancy strategy in naming, but the naming task appears to be rather insensitive to manipulations of relatedness pro-

This research was supported by Grant A79532361 to the first two authors. Correspondence concerning this article should be addressed to M. S. Humphreys, Key Centre for Human Factors and Applied Cognitive Psychology, University of Queensland, QLD 4072, Australia (email: m.humphreys@humanfactors.uq.edu.au). portion, perhaps because, in contrast to the situation in the lexical decision task, response times with the expectancy strategy may not be substantially faster than response times without the strategy. Alternatively, subjects may rely on expectancy strategies only when the predictability of the target is very high. Investigation of expectancy strategies in word recognition and reading of text would be facilitated by an additional source of evidence that allows diagnosis of expectancy strategy use. The present study focused on the use of postpriming memory measures for this purpose. Memory indices of prime and target processing may assist in distinguishing prospective, expectancybased effects from retrospective strategies instigated after onset of the target (see the review by Neely, 1991).

The experiment consisted of an associative priming manipulation in a word-naming task, followed by a recognition memory test conducted after the priming phase. As will be described below, the priming task was augmented by the inclusion of a misprimed condition in which an associatively related target was replaced by an orthographically and generally phonologically similar foil (see, Norris, 1984; O'Connor \& Forster, 1981). This procedure allowed for a more comprehensive assessment of expectancy-based strategies. Importantly, it additionally provided information about the nature of the subjects' memories of the words generated as an expectation during the priming task. Indeed, although the experiment was motivated by questions about strategic processing in reading, the most intriguing results are those that relate to questions about memory representations.

In the priming phase of the experiment, the subjects silently read primes and overtly named targets. In order to induce a strong expectation, we used time pressure in conjunction with a high proportion of related prime-target pairs. In the high-proportion group, the majority of primetarget pairs were associatively related. In the lowproportion group, the majority of prime-target pairs were unrelated. In addition, in both groups, the targets 
were rapidly presented. The rapid presentation of targets makes our task a bit of a hybrid between a naming and a perceptual identification task. It is still a naming task in that subjects report clearly seeing the targets and naming accuracy is still high.

In both groups, three types of targets were used. Some targets were associates of the prime (primed targets); there were other targets where the associate was replaced by an orthographically and generally phonologically similar but semantically dissimilar foil (misprimed target) and other targets in which the target was a foil from another pair and thus was unrelated to the associate of the prime (unprimed target). For example, the misprimed target dart would be paired with the prime soll. Thus, in this condition, the highproportion subjects will generate the expectation that the target that will follow the prime SOIL is going to be dirt. At approximately the same time, they read dart, a word that is visually (orthographically) similar to their expectation.

With an unprimed target, there are two relevant primetarget pairs (LENGTH-honey and OFFICE-witch). Thus, with unprimed targets, the high-proportion subjects generate the expectation that the target that will follow the prime LENGTH is going to be width. They also read the word witch. The crucial difference between the two conditions is that the two activities (generating the expectation and reading the orthographically similar word) occur almost simultaneously with misprimed targets. However, with unprimed targets these two activities are separated by the presentation of other prime-target pairs.

The recognition test consisted of words presented as targets in the priming phase that were associates of their prime (primed targets), unpresented words that were associates of the prime of a misprimed target (misprimed lures), unpresented words that were associates of the prime of an unprimed target (unprimed lures), and words that were associates of a prime that was not present in the priming phase (new lures). Examples of the study/priming and test/recognition conditions are presented in Table 1 .

If subjects are more likely to generate an expectation in the high-proportion condition, there is a straightforward prediction about the hit rates (HRs) in the two condi-

Table 1

Examples of the Relationships Between Items Presented at Study (the Priming Task) and the Test Items on the Recognition Test

\begin{tabular}{|c|c|c|c|}
\hline $\begin{array}{l}\text { Priming } \\
\text { Condition }\end{array}$ & $\begin{array}{c}\text { Presented } \\
\text { During Priming }\end{array}$ & $\begin{array}{c}\text { Recognition } \\
\text { Condition }\end{array}$ & Test Item \\
\hline $\begin{array}{l}\text { Primed target } \\
\text { Misprimed target } \\
\text { Unprimed target }\end{array}$ & $\begin{array}{l}\text { DOCTOR-nurse } \\
\text { SOIL-dart } \\
\text { LENGTH-honey } \\
\text { OFFICE-witch } \\
\quad \text { - }\end{array}$ & $\begin{array}{l}\text { primed target } \\
\text { misprimed lure } \\
\text { unprimed lure } \\
\text { new lure }\end{array}$ & $\begin{array}{l}\text { nurse } \\
\text { dirt } \\
\text { width }\end{array}$ \\
\hline
\end{tabular}

Note-The names for the study/priming conditions refer to the target that was presented at study, and the names for the test/recognition conditions refer to the word that was tested for recognition. The primeassociate-foil triples used to construct these examples are soIL-dirtdart, DOCTOR-nurse-purse; LENGTH-width-witch, OFFICE-work-word, and CHAIR-table-fable. tions. The suggestion that naming may be accomplished via grapheme-phoneme correspondence rules without accessing the lexicon (Andrews, 1989; Balota \& Chumbley, 1985) provides one basis for expecting that the HR will be lower in the low-proportion condition, in which such a nonsemantic naming process is more likely. More generally, any model that allows subjects to go from an orthographic representation to a phonemic representation without fully activating or accessing the meaning of the word could make the same prediction. That is, it seems plausible that naming a word via a grapheme-to-phoneme route would correspond to the shallow or perceptual processing tasks that are known to produce poor episodic memories (Craik \& Lockhart, 1972). In contrast, naming a word via a route that accesses the lexicon and/or meaning might be similar to the deep or semantic processing tasks that are known to produce good episodic memories.

The preceding intuitive justification for expecting an increase in the HR can be formalized within a connectionist model. For example, this kind of poor learning would occur with sparse representations (most of the units are inactive) if the input and the output layers of a connectionist model were only partially connected. In such a model, learning would occur only when an active unit at the input layer was connected to an active unit at the output layer, and the probability of this happening would increase as the sparseness decreased at the two layers (see Dennis \& Humphreys, 2001, for a model of recognition that could be adapted to incorporate such a learning mechanism). A sparse representation might tend to occur with a novel word (Chalmers \& Humphreys, 1998) or with a word for which only the perceptual features have been encoded.

It also seemed possible that false alarm rates (FARs) to misprimed lures and unprimed lures would be higher in the high- than in the low-proportion condition. This elevation in the FAR could occur if the generation of an expectation corresponds to the implicit associative response postulated by Underwood (1965). That is, under this assumption, the act of generation produces a memory for the generated but unpresented word. Alternatively, at test, subjects in the high-proportion condition might realize that there were many related prime-target pairs in the list. Under these conditions, they might adopt a recall strategy in order to recognize (Humphreys \& Bowyer, 1981). That is, subjects may identify a word as old if they can recall the word's associate. The important point about these two alternatives is that they predict that the FARs in the highproportion condition will be elevated for both the misprimed lures and the unprimed lures. That is, neither alternative assigns any importance to the nearly simultaneous generation of an expectation and the reading of the orthographically related word that occurs for misprimed lures, as compared with the separation of these two events with unprimed lures.

However, it seems possible that the nearly simultaneous generation of an expectation and the reading of an orthographically similar word could be important. For ex- 
ample, with distributed representations, there does not seem to be any impediment, on at least some trials, to storing an episodic memory that combines the semantic information generated and the sensory information derived from reading the target. To illustrate, with a misprimed lure (dirt), there is one relevant memory (the memory created when the subject formed the expectation that dirt was going to be presented and read dart). We represent the semantic expectation (DIRT) and sensory input (dart) as vectors. We then superimpose the meaning vector DIRT and the perceptual vector dart and associate them with a context vector. At test, we compare the probe vector (in this example, it contains both the meaning component, DIRT, and the perceptual component, dirt) with the memory vector (Dennis \& Humphreys, 2001; Shiffrin \& Steyvers, 1997). Both the meaning component and the perceptual component of the probe will help to produce an elevated FAR. The meaning component of the probe contributes because it is the same as the meaning component of the memory, and the perceptual component contributes because it is similar to the perceptual component of the memory. ${ }^{1}$

With an unprimed lure (width), there are two relevant study pairs (e.g., LENGTH-honey and OFFICE-witch), so we consider the memories formed from both pairs. In the high-proportion condition, one of these memories, on at least some trials, would be the association of the superimposed meaning vector WIDTH and perceptual vector honey with the context vector. The other would be the association of the superimposed meaning vector WORK and perceptual vector witch with the context vector. At test we would use the meaning component, WIDTH, and the perceptual component, width, of the probe to compare with memory. In this case, we do not expect that having both a meaning component and a perceptual component in memory would contribute to an elevated FAR, because one component always mismatches. That is, the meaning component of the probe vector (WIDTH) will mismatch the meaning component of the WORK + witch vector, and the perceptual component of the probe vector (width) will mismatch the perceptual component of the WIDTH + honey vector. In fact, in some models, a mismatching component would be expected to depress the FAR relative to the low-proportion condition, where we would assume that the relevant memories contain only the perceptual component (honey or witch; see note 1).

\section{METHOD}

\section{Subjects}

The subjects were 96 introductory psychology students at the University of Queensland. This was the second of two short experiments in which these subjects participated in order to earn $1 \mathrm{~h}$ of research credit. The first experiment, which immediately preceded this one, was a sentence-priming experiment conducted by the same experimenter that was otherwise unrelated. The subjects were randomly assigned to one of the two proportion conditions, with the constraint that an equal number of subjects was assigned to each of the two conditions.

\section{Lists and Materials}

The University of South Florida Norms (Nelson, McEvoy, \& Schreiber, 1994) provided 64 pairs of words collected in free association tests. The mean associative strength between the prime and its associate, indexed by the proportion of the norm sample giving the target as their response to the prime, was $28 \%$. The primes ranged in length from 3 to 8 letters (mean $=4.9)$ and in frequency of occurrence per million from 6 to $109($ mean $=56.3)$. The associates ranged in length from 4 to 5 letters (mean $=4.5)$ and in frequency from 2 to 348 (mean $=121.5)$. For each associate, an orthographically similar word, sharing with the target all but one or two letters in position, was selected as an orthographically similar foil. Letter substitutions were distributed over the letter positions, with all except position 5 being represented in at least $20 \%$ of target-foil pairs. Foils had at least some degree of phonological similarity with the associate but were semantically dissimilar; for example, the prime soIL had associate dirt and foil dart. Additional examples are DOCTOR-nurse, purse; LENGTH-width, witch; CHAIR-table, fable, and OFFICE-work, word. It was not possible to match foil and associate frequency, and the foils were somewhat lower in frequency than were the corresponding associates. The foils ranged in frequency from 0 to 313 (mean $=38.7$ ). The stimulus words served as primes in the study/priming conditions. The response words and the orthographic foils served as targets in the study/priming conditions. Only the response words served as test items in the test/recognition conditions. An additional set of 20 strong associates were used as filler prime-target pairs for manipulation of the relatedness proportion. The associated filler pairs were randomly re-paired to produce a second set of unrelated filler pairs.

The 64 experimental prime-associate-foil triples were randomly divided into four subsets of 16 triples each. Subsets were assigned to one of the three target types (primed, misprimed, and unprimed) for the priming task and to one of four recognition tests types (primed target, misprimed lure, unprimed lure, and new lure) for the recognition task. Eight priming task lists of 68 pairs were constructed by counterbalancing the assignment of items in the four subsets of triples to the priming and recognition conditions and then adding either the 20 associated filler pairs or the 20 unrelated filler pairs, according to relatedness proportion group. The result was that each subset of 16 served equally often in each of the priming and recognition conditions. The order of presentation of the 68 prime-target pairs in each priming list was randomized. The test list consisted of the 64 associates and was individually randomized for each subject.

\section{Procedure}

After completing the prior experiment, the subjects were informed that the next experiment had two phases and that neither phase was related to the experiment they had just completed. The subjects were informed that the first phase would measure the speed and accuracy which they could read words. They were informed that a warning signal $(+)$ would first appear in the center of their computer screen and that, when the warning signal disappeared, it would be replaced by a word presented in uppercase, which in turn would be replaced by a word in lowercase, which in turn would be replaced by a string of letters. They were instructed to read the uppercase word silently to themselves and then to read the lowercase word out loud as fast as they could.

Ten prime-target pairs were presented as practice trials. In the lowproportion condition these were unrelated, and in the high-proportion condition they consisted of six related and four unrelated pairs. During practice, the warning signal appeared for $500 \mathrm{msec}$. The uppercase word also appeared for $500 \mathrm{msec}$, and it was followed by a $250-\mathrm{msec}$ blank period. Then the lowercase word appeared for $98 \mathrm{msec}$, after which it was replaced by a letter mask. The letter mask stayed on until the subject responded and the experimenter pushed a button after recording the response. The same timing parameters were used for the experimental materials during the priming task. The same pa- 
rameters were also used for the filler materials during the priming task, except that, with these materials, the lowercase words were presented only for $56 \mathrm{msec}$. Naming latencies were recorded with a voice key. The faster presentation rate for the filler items was intended to increase the perceived need for rapid processing in our subjects without reducing the clarity with which the critical words were presented. The experimenter was the third author, who is linguistically trained. She followed a printed list of prime-target pairs to facilitate scoring vocal responses during the priming task. A small number of responses was unclear and was repeated at the experimenter's request.

After completing the priming task, the subjects were instructed that they were going to be asked to recognize the lowercase words they had named during the priming task. The to-be-recognized words were then presented one at a time. The subjects responded using the computer keyboard to implement a 6-point rating scale that was displayed with labels on the screen. Scores in the range of 1-3 were for new responses, with 1 for sure and 3 for unsure, and scores from 4 to 6 signified old, with 4 for unsure and 6 for sure. The next word was presented as soon as the subject responded.

\section{RESULTS}

The accuracy and latency of responding during the priming task is presented for the targets in the primed, misprimed, and unprimed conditions for both proportion groups in Table 2. The standard error for each condition (based on the between-subjects variability) is presented in parentheses. In preparing the latency data, a mean was calculated for each person, excluding naming errors, responses shorter than $200 \mathrm{msec}$ or longer than $2,000 \mathrm{msec}$, and microphone failures. Response times not within three standard deviations of the subject's mean were also deleted. Across all conditions, on average, $1.8 \%$ of the responses were excluded for reasons other than naming errors. In most cases, in Table 2, significance can be determined by an inspection of standard errors. That is, any difference greater than two standard errors should be considered significant. In those few cases in which significance may be in doubt, we also report analyses of variance conducted on items in order to complement the subject-based effects that can be derived from Table 2. In all analyses, $p<.05$ is considered significant.

In both proportion groups, the subjects were more accurate and faster in responding to targets in the primed condition than to targets in the misprimed and unprimed conditions. These differences probably result from both priming and the fact that the targets in the primed condition were somewhat higher in frequency than were the tar- gets in the other two conditions. In addition, the performance of the high-proportion group on the misprimed targets was slightly suppressed, relative to performance on the same targets in the low-proportion group and relative to performance on the unprimed targets in both proportion groups. To examine this effect further, we conducted an items analysis. The difference in accuracy within the highproportion condition between the misprimed and unprimed targets was marginally significant $[F(1,63)=3.99$, $\left.M S_{\mathrm{e}}=0.019, p=.05\right]$.

We also looked at the probability that, during the priming task, a misprimed target or an unprimed target was falsely identified as the associate of the prime. Note that with unprimed targets this is purely a visual confusion error (e.g., dart is confused with dirt after the prime SLOW), but in the misprimed condition this may represent the joint effects of an expectation generated in response to the prime SOIL and a visual confusion between dart and dirt. In the high-proportion group, these probabilities were .069 and .002 for the misprimed and unprimed targets, respectively. In the low-proportion group, these probabilities were .036 and .005 for the misprimed and unprimed targets, respectively. The intrusion of associates with the misprimed targets was observed for 28 of the 64 word triples and was not restricted to items where it was likely that the vocal responses were ambiguous or a blend of the foil and associates. Common errors were sleep for steep, brain for braid, and king for kind.

Although subjects made more overt errors with misprimed targets in the high-proportion group, there was no evidence that they were more likely to make covert errors that were corrected before an overt response was made. That is, the average latency for misprimed targets was only $6 \mathrm{msec}$ longer for the high-proportion subjects than it was for the low-proportion subjects. This difference is nonsignificant, since it is less than one standard error.

The recognition results are based on correctly identified items. The rating task had been included as a hedge against floor and ceiling effects. Since these were not present, it was decided to analyze only the probability of identifying the item as old (a rating of 4 or higher). The results are presented in Table 3. For both groups, the HR (the probability of an old response to primed targets) is well above the three FARs (the probabilities of old responses to misprimed lures, unprimed lures, and new lures). Furthermore, the FARs to the misprimed lures and unprimed lures

Table 2

Accuracy and Latency Results in the Priming Task for Primed Targets, Misprimed Targets, and Unprimed

Targets for Both the High- and the Low-Proportion Groups Run Under Time Pressure and for an Additional High-Proportion Group Under No Time Pressure

\begin{tabular}{|c|c|c|c|c|c|c|c|c|c|c|c|c|}
\hline \multirow[b]{4}{*}{ Priming Condition } & \multicolumn{8}{|c|}{ Time Pressure } & & & & \\
\hline & \multicolumn{4}{|c|}{ High Proportion } & \multicolumn{4}{|c|}{ Low Proportion } & \multicolumn{4}{|c|}{ No Time Pressure } \\
\hline & \multicolumn{2}{|c|}{ Accuracy } & \multicolumn{2}{|c|}{ Latency } & \multicolumn{2}{|c|}{ Accuracy } & \multicolumn{2}{|c|}{ Latency } & \multicolumn{2}{|c|}{ Accuracy } & \multicolumn{2}{|c|}{ Latency } \\
\hline & $M$ & $S E$ & $M$ & $S E$ & $M$ & $S E$ & $M$ & $S E$ & $M$ & $S E$ & $M$ & $S E$ \\
\hline Primed target & .98 & .006 & 507 & 10.7 & .99 & .004 & 523 & 12.8 & .99 & .004 & 464 & 6.2 \\
\hline Misprimed target & .85 & .015 & 558 & 16.5 & .90 & .016 & 552 & 15.8 & .97 & .006 & 481 & 7.0 \\
\hline Unprimed target & .89 & .015 & 560 & 14.5 & .90 & .017 & 559 & 14.7 & .99 & .005 & 481 & 6.7 \\
\hline
\end{tabular}


Table 3

Probability of an Old Response on the Recognition Test for Primed Targets, Misprimed Lures, Unprimed Lures, and New Lures for Both the High- and the Low-Proportion Groups That Performed the Naming Task Under Time Pressure and for an Additional High-Proportion Group Under No Time Pressure

\begin{tabular}{|c|c|c|c|c|c|c|}
\hline \multirow{3}{*}{$\begin{array}{l}\text { Recognition } \\
\text { Condition }\end{array}$} & \multicolumn{4}{|c|}{ Time Pressure } & & \\
\hline & \multicolumn{2}{|c|}{$\begin{array}{c}\text { High } \\
\text { Proportion }\end{array}$} & \multicolumn{2}{|c|}{$\begin{array}{c}\text { Low } \\
\text { Proportion }\end{array}$} & \multicolumn{2}{|c|}{$\begin{array}{l}\text { No Time } \\
\text { Pressure }\end{array}$} \\
\hline & $M$ & $S E$ & $M$ & $S E$ & $M$ & $S E$ \\
\hline Primed target & .75 & .021 & .66 & .026 & .64 & .030 \\
\hline Misprimed lure & .37 & .029 & .31 & .026 & .32 & .031 \\
\hline Unprimed lure & .28 & .024 & .32 & .028 & .30 & .031 \\
\hline New lure & .25 & .025 & .24 & .027 & .25 & .030 \\
\hline
\end{tabular}

are generally higher than the FAR to the new lures. This small enhancement of the FAR rate owing to the presence of an associatively related and/or a physically related item in the study list is consistent with the generally observed magnitude of these effects (Underwood \& Humphreys, 1979).

We were successful in inducing subjects to adopt an expectancy strategy, since the HR was more than two standard errors larger in the high-proportion group than in the low-proportion group. An items analysis confirmed this result $\left[F(1,63)=12.95, M S_{\mathrm{e}}=0.029\right]$. This occurred despite the fact that, during the priming phase, there was no significant difference in the latencies for the primed targets between the high- and the low-proportion groups.

The expectancy manipulation also elevated the FAR. However, this effect was confined to the misprimed lures where the increase was greater than two standard errors. An items analysis of the interaction of the factors associate type (misprimed vs. unprimed) and relatedness proportion group on the number of lures falsely recognized was also significant $\left[F(1,63)=4.50, M S_{\mathrm{e}}=0.028\right]$, confirming this finding.

In order to eliminate the possibility that this interaction between associate type and relatedness proportion resulted from incorrect recording of responses (e.g., a subject incorrectly says dirt but was "misheard" by the experimenter as saying dart), we looked at the phonological similarity between each associate and its corresponding foil. When the item set was divided on this basis, there was no significant difference $(F<1)$ in the mean proportion of false alarms for the misprimed lures for the 28 associates judged to be less phonologically similar to their foils (e.g., there-theme, power-rower, and bird-bind; mean FAR = .36) and the 36 associates judged to be more similar to their foils (e.g., sting-sling, place-plate, and brain-braid; mean FAR $=.40)$. This small difference between the FARs for the associates less phonologically similar and more phonologically similar to their foils provides no support for the mishearing hypothesis, since it was also observed in the low-proportion group, where the means were .28 for associates less phonologically similar and .33 for associates more phonologically similar to their foils $(F<1)$.
Prior to running this experiment, a high-proportion group had been run without the time pressure during the priming task, although all other procedures were the same. The 48 subjects were similar to those used in the present experiment, and all of the materials were the same. These results are also shown in Tables 2 and 3. As can be seen in Table 3, the results for the high-proportion group without time pressure are almost identical to the results for the low-proportion group with time pressure. In particular, there was no difference in the HRs between the highproportion condition without time pressure and the lowproportion condition with time pressure. Thus, there was no indication that subjects in the high-proportion condition without time pressure adopted an expectancy strategy. Similarly, there was no difference in the accuracy or latency of responding to the misprimed and unprimed targets during the priming task (Table 2). This betweenexperiments comparison suggests that the subjects did not use an expectancy strategy in the absence of time pressure. It also suggests that the elevated FAR with misprimed lures was the result of the adoption of the expectancy strategy. It is also an indication of the stability of our recognition results, which is attributable to the large sample sizes and, possibly, to the control exerted over strategies by the priming task.

\section{DISCUSSION}

If we focus exclusively on the priming results, the usual conclusion that expectancy priming can be present but is hard to find with a naming task holds (Burt et al., 1996; Keefe \& Neely, 1990). The identification of misprimed targets appears to have been depressed in the high-proportion group, relative to the identification of unprimed targets in the same group. However, despite the very large sample size, this difference was only marginally significant. In addition, presenting a target item that is physically similar to an expected target (misprimed target) increased the probability that the presented target would be falsely identified as the expected target. These findings are evidence for expectancy priming. However, the absolute magnitude of these differences was quite small and could easily be dismissed, especially with more normal sample sizes. That is, it would be quite possible to look at these data and conclude that there was evidence only for an automatic priming effect.

This standard conclusion changes when we include the recognition results. Here, the expectancy manipulationincreased the probability of saying old when the expectancy was at least partially congruent with the presented target. That is, it increased the HR when the target on the priming phase was completely congruent with the expectation or an element in the expectation, and it increased the FAR to associates of the prime (the expectation) where the target in the priming phase was orthographically and phonologically similar, but not semantically similar. Thus, recognition performance provided a more sensitive indicator that the subjects had changed the way they were naming the target than did the standard priming results. 
In other words, latency is an insensitive indicator of the presence of expectancy priming in word naming.

The elevated FAR in the high-proportion group for the associates of the prime where the target was orthographically similar (misprimed lure) is also evidence for a false memory. Although it is a small effect, it is almost certainly reliable. That is, it was significant by both a subjects and an items analysis. In addition, it was essentially unchanged when we grouped the associates on the basis of how phonologically similar they were to the foils. Finally, the large sample size and, possibly, the tight control exerted over learning strategies by the priming task produced very consistent data.

This false memory cannot be attributed to the similarity between the memory probe and the items in memory, because this similarity is held constant across misprimed and unprimed lures. It also cannot be attributed to an increase in the likelihood of implicitly producing an associate of the prime in the high-proportion condition or to the use of a recall strategy in recognition, since these effects would also occur as often or as readily with unprimed lures. Finally, it cannot be attributed to a generation process that occurs after the target was named. Because the target stayed in view until a response was made, such a process would have been easier to adopt in the high proportion group without time pressure than in the high-proportion group with time pressure.

Instead, the false memory results from the use of an expectancy strategy. In turn, the expectancy strategy results in the nearly simultaneous occurrence of semantic information (from the generation of an expectation) and sensory information (from the reading of the foil that is orthographically and phonologically similar to the expectation). The result is a memory for a real episode (the nearly simultaneous activation of the expectation and the sensory information), but it is a memory for an entity that does not exist in the subject's semantic memory. That is, it is a blend episodic memory that has been created from two different semantic entities.

The assumption that words are represented in a distributed fashion is still highly controversial, although there is now sufficient evidence to require that it be taken seriously. For example, Tehan and Humphreys (1998) used a short term interference paradigm where a List 1 item $(d o g)$ and a List 2 item (cat) were both subsumed under the same cue (ANIMAL). They were able to increase the recall of the to-be-forgotten item in List 1 by presenting its phonemes in three different (dart, mop, and fig) List 2 words. Similarly, Humphreys, Tehan, O'Shea, and Bolland (2000) were able to show how an interaction between two highly similar items subsumed under the same cue could be explained by assuming distributed representations but was difficult to explain if representations were not distributed. The present demonstration of a false memory follows in this tradition. This finding is relatively easy to explain if we assume distributed representations and relatively difficult to explain if we assume local or symbolic representations.
Although an explanation using local or symbolic representations cannot be ruled out, such an explanation needs to take the following findings into consideration. First, in the priming data, there was no evidence for competition or conflict with the misprimed targets in the high-proportion group, relative to the same targets in the low-proportion group. Thus, there is no evidence that, with misprimed targets, the subjects in the high-proportion group covertly produced the associate of the prime and then rejected it without overtly producing it. Second, although the subjects in the high-proportion group were clearly generating expectations, this generation in itself did not increase the FAR. That is, the FAR for associates in the unprimed condition was no higher in the high-proportion group with time pressure than it was in the low-proportion group with time pressure. Thus, there is no evidence in this situation to suggest that a subthreshold level of activation will produce a memory. In addition, future research should look at the possibility that the FAR for unprimed lures and misprimed targets will be depressed in the high-proportion condition, relative to the same items in the low-proportion condition. The effect here may be so small that it is unlikely to be significant in any one study. However, if such an effect were found over a series of studies it would be very difficult to explain using symbolic representations.

The important point is not whether we are able to conclusively prove that either of these starting points (distributed vs. separate representations) is correct but whether the starting point is leading to new findings. In this regard, we believe that by starting with the assumption that representations are distributed, we are generating important new findings.

\section{REFERENCES}

ANDREws, S. (1989). Frequency and neighborhood effects on lexical access: Activation or search? Journal of Experimental Psychology: Learning, Memory, \& Cognition, 15, 802-814.

Balota, D. A., \& Chumbley, J. I. (1985). The locus of word frequency effects in the pronunciation task: Lexical access and/or production? Journal of Memory \& Language, 24, 89-106.

Burt, J. S., Mardle, L. I., \& Humphreys, M. S. (1996). Expectancybased associative and identity priming in pronunciation. Australian Journal of Psychology, 48, 64-74.

Burt, J. S., Walker, M. B., Humphreys, M. S., \& Tehan, G. (1993). Associative priming in perceptual identification: Effects of primeprocessing requirements. Memory \& Cognition, 21, 125-137.

Chalmers, K. A., \& Humphreys, M. S. (1998). Role of generalized and episode specific memories in the word frequency effect in recognition. Journal of Experimental Psychology: Learning, Memory, \& Cognition, 24, 610-632.

Craik, F. I. M., \& Lockhart, R. S. (1972). Levels of processing: A framework for memory research. Journal of Verbal Learning \& Verbal Behavior, 11, 671-684.

Dennis, S., \& Humphreys, M. S. (2001). A context noise model of episodic word recognition. Psychological Review, 108, 452-478.

Humphreys, M. S., \& Bowyer, P. A. (1981). Cued recall and cue recognition: How similar are the processes? American Journal of Psychology, 94, 65-84.

Humphreys, M. S., Tehan, G., O’Shea, A., \& Bolland, S. W. (2000). Target similarity effects: Support for the parallel distributed processing assumptions. Memory \& Cognition, 28, 798-811. 
Keefe, D. E., \& Neely, J. H. (1990). Semantic priming in the pronunciation task: The role of prospective prime-generated expectancies. Memory \& Cognition, 18, 289-298.

NeEley, J. H. (1991). Semantic priming effects in visual word recognition: A selective review of current findings and theories. In D. Besner \& G. W. Humphreys (Eds.), Basic processes in reading: Visual word recognition (pp. 264-336). Hillsdale, NJ: Erlbaum.

Nelson, D. L., McEvoy, C. L., \& Schreiber, T. (1994). The University of South Florida word association, rhyme and word fragment norms. Unpublished manuscript.

NorRIS, D. (1984). The mispriming effect: Evidence of an orthographic check in the lexical decision task. Memory \& Cognition, 12, 470-476.

O'CONNOR, R. E., \& Forster, K. I. (1981). Criterion bias and search sequence bias in word recognition. Memory \& Cognition, 9, 78-92.

Seidenberg, M. S., Waters, G. S., SAnders, M., \& Langer, P. (1984). Pre- and postlexical loci of contextual effects on word recognition. Memory \& Cognition, 12, 315-328.

Shiffrin, R. M., \& Steyvers, M. (1997). A model for recognition memory: REM-retrieving effectively from memory. Psychonomic Bulletin \& Review, 4, 145-166.

Tehan, G., \& Humphreys, M. S. (1998). Creating proactive interference in immediate recall: Building a DOG from a DART, a MOP, and a FIG. Memory \& Cognition, 26, 477-489.

Tweedy, J. R., Lapinski, R. H., \& Schvaneveldt, R. W. (1977). Semantic-context effects on word recognition: Influence of varying the proportion of items presented in an appropriate context. Memory \& Cognition, 5, 84-99.

UNDERWOOD, B. J. (1965). False recognition produced by implicit verbal responses. Journal of Experimental Psychology, 70, 122-129.
Underwood, B. J., \& Humphreys, M. S. (1979). Context change and the role of meaning in word recognition. American Journal of Psychology, 92, 577-609.

\section{NOTE}

1. Shiffrin and Steyvers's (1997) model uses both positive and negative information in reaching a recognition decision. Thus, it should predict that a mismatch (negative evidence) in either the semantic or the perceptual component would reduce the FAR, relative to a condition in which that component was not present. Dennis and Humphreys's (2001) model may also be able to make the same prediction, for related reasons. Note that a comparison between the recognition of misprimed lures and that of misprimed targets would not provide a test of the use of negative evidence, because this comparison is confounded by the use of different items and the presence of both a matching and a partially matching component with misprimed lures. However, a recognition test for misprimed and unprimed targets in the high and low relatedness proportion conditions would provide information on the role of negative information. That is, if the recognition of the targets in the high-expectancy condition were poorer than the recognition of the same targets in the low-proportion condition, it would support the use of negative information. We did not, however, test either the misprimed or the unprimed targets, because it seems likely that any effect that is due to the use of negative information will be small.

(Manuscript received October 18, 1999; revision accepted for publication April 6, 2001.) 